\title{
Correspondence
}

\section{Requested retraction of six studies on the PK/PD and tolerability of fospropofol}

Michel M.R.F. Struys, Jörg Fechner, Jürgen Schüttler and Helmut Schwilden

From the Department of Anesthesiology, University of Groningen, Groningen, The Netherlands and Professor, Department of Anesthesia, Ghent University, Gent (MMRFS), Belgium, Department of Anesthesiology, University ErlangenNuremberg, Erlangen (JF, JS), Germany and Section of Experimental Anesthesiology, University Erlangen-Nuremberg, Erlangen (HS), Germany

Correspondence to Michel M.R.F. Struys, MD, PhD, Professor and Chair, Department of Anesthesiology, University of Groningen, Groningen, The Netherlands and Professor, Department of Anesthesia, Ghent University, Gent, Belgium

Tel: +310 5036155 19; fax: +310 503613763 ;

e-mail: m.m.r.f.struys@anest.umcg.nl

\section{Dear Drs Eisenach, Shafer and Tramèr,}

As described in a Letter to the Editor, published in Anesthesiology, Anesthesia \& Analgesia and the European Journal of Anaesthesiology, ${ }^{1-3}$ an analytical propofol assay inaccuracy was discovered after all six initial studies on the PK/PD and tolerability of fospropofol had been published. ${ }^{4-9}$ This assay inaccuracy makes the measured propofol plasma concentrations in these previously published studies unreliable.

All six affected studies were Phase I and II studies sponsored by a pharmaceutical company (Guilford Pharma, Baltimore, MD, USA and later MGI Pharma, Baltimore, MD, USA) and were performed in two independent academic-based phase I centers in Gent, Belgium and Erlangen, Germany. Due to the stage of the drug testing, the study drugs were made available by the initial Sponsor. As described previously, ${ }^{1-3}$ the Sponsor developed and validated a specific propofol assay. Both academic centers had no influence on the choice of methodology for sample handling and chemical analysis. For all six studies ${ }^{4-9}$ assays were performed at an external laboratory (MDS Pharma Services, Montreal, Canada) as per Sponsor decision. Finally, the original publications were co-authored by both academic and Sponsor-based investigators.

In a Letter to the Editor ${ }^{1-3}$ the initial owner of the drug (MGI Pharma, Baltimore, MD, USA- not affiliated the academic centers from the original studies) declared that additional studies were planned using an appropriate assay to describe the pharmacokinetics and pharmacodynamics of fospropofol in healthy volunteers and patients. They stated their intent to publish these results shortly, along with an estimate of the degree of error from the previously published studies reporting results using the old assay. In the "In Reply" response, you the Editors-in-
Chief of Anesthesiology, Anesthesia \& Analgesia and the European Journal of Anaesthesiology requested a publication within the next 12 months validating the new assay, analyzing the likely error and bias in each of the six articles in question, and determining how the error and its correction would influence the conclusions.

Due primarily to transfer of ownership of the drug to another pharmaceutical company in mid 2009 (Eisai, Woodcliff Lake, NJ, USA), the planning of studies was delayed. As a result and although requested by the academic investigators immediately after the publication of the Letter to the Editor, ${ }^{1-3}$ the investigators from the original studies were not able to reanalyze the PK/PD of fospropofol in human volunteers within the deadline of 12 months given by the Editors-in-Chief. As such, we, the undersigned corresponding and senior authors from the six original papers, in the name of all co-authors, request that the papers in question which provide flawed $\mathrm{PK} / \mathrm{PD}$ data be retracted. We regret that we are unable to successfully resolve the problem within the given timeframe.

\section{References}

1 Shah A, Mistry B, Gibiansky E, Gibiansky L. Fospropofol assay issues and impact on pharmacokinetic and pharmacodynamic evaluation. Anesthesiology 2008; 109:937; discussion.

2 Shah A, Mistry B, Gibiansky E, Gibiansky L. Fospropofol assay issues and impact on pharmacokinetic and pharmacodynamic evaluation. Anesth Analg 2009; 108:382.

3 Shah A, Mistry B, Gibiansky E, Gibiansky L. Fospropofol assay issues and impact on pharmacokinetic and pharmacodynamic evaluation. Eur $J$ Anaesthesiol 2009; 26:81.

4 Fechner J, Ihmsen H, Hatterscheid D, Schiessl C, Vornov JJ, Burak E, Schwilden H, Schuttler J. Pharmacokinetics and clinical pharmacodynamics of the new propofol prodrug GPI 15715 in volunteers. Anesthesiology 2003; 99:303-313.

5 Fechner J, Ihmsen H, Hatterscheid D, Jeleazcov C, Schiessl C, Vornov JJ, Schwilden $\mathrm{H}$, Schuttler J. Comparative pharmacokinetics and pharmacodynamics of the new propofol prodrug GPI 15715 and propofol emulsion. Anesthesiology 2004; 101:626-639.

6 Fechner J, Ihmsen H, Schiessl C, Jeleazcov C, Vornov JJ, Schwilden H, Schuttler J. Sedation with GPI 15715, a water-soluble prodrug of propofol, using target-controlled infusion in volunteers. Anesth Analg 2005; 100:701-706.

7 Gibiansky E, Struys MMRF, Gibiansky L, Vanluchene AL, Vornov J, Mortier EP, Burak E, Van Burtel L. AQUAVAN Injection, a water-soluble prodrug of propofol as bolus injection: a Phase I dose escalation comparison with DIPRIVAN. Part 1: pharmacokinetics. Anesthesiology 2005; 103:718729.

8 Struys MMRF, Vanluchene ALG, Gibiansky E, Gibiansky L, Vornov J, Mortier EP, Van Bortel L. AQUAVAN, a water-soluble prodrug of propofol as a bolus injection: A Phase I dose escalation comparison with DIPRIVAN (part 2): pharmacodynamics and safety. Anesthesiology 2005; 103:730-743.

9 Schywalsky M, Ihmsen H, Tzabazis A, Fechner J, Burak E, Voronov J, Schwilden $\mathrm{H}$. Pharmacokinetics and pharmacodynamics of the new propofol prodrug GPI 15715 in rats. Eur J Anaesthesio/ 2003; 20:182190. 


\section{Trainee anaesthetists' attitudes to error, safety and the law}

Simon J. Mercer, Michael J. Moneypenny and Arpan Guha

Cheshire and Merseyside Simulation Centre, University Hospital Aintree, Liverpool, UK

Correspondence to Simon Jude Mercer, MB ChB PgDipMEd FRCA, Cheshire and Merseyside Simulation Centre, University Hospital Aintree, Longmoor Lane, Liverpool L9 7AL, UK

Tel: +44 151529 8210; e-mail: arpan@simulationcentre.com

Received 7 July 2009 Accepted 16 July 2009

Editor,

We read with interest the article by White et al. [1] and would suggest that many of the issues raised could be dealt with by attending a team resource management (TRM) course using a high-fidelity human patient simulator. As the authors point out, in order to reduce legal liability and improve patient safety, professional guidelines must be adhered to. A recent study [2] has concluded that the use of guidelines by a professional group is best understood as a product of the group's social norms, work practices and organizational culture. A TRM course concentrating on the human factors required to follow guidelines such as communication, leadership and teamwork may be of benefit in promoting adherence. Anaesthetists who have trained on a high-fidelity anaesthesia simulator previously have been shown to respond more quickly and deviate less from accepted guidelines [3].

Procedural errors accounted for approximately a third of worst errors made by trainees in this study. Attendance on simulation courses provides an opportunity to practise skills and procedures. Whether this is using a part-task trainer, a computer-based system or an integrated simulator, evidence from cognitive psychological research on expertise has reported that to make the transition to expert status, many hours of practice are required [4] and that deliberate practice is better than simple unstructured practice [5]. TRM skills that are learnt in the context of simulated anaesthetic emergencies are retained and are transferable across the breadth of all clinical activities [6]. Simulation offers the trainee the opportunity to determine their own learning objectives by setting the agenda to concentrate on whole procedures without having to concentrate specifically on patient care [7] in a safe environment.

Exploration of the medicolegal aspects of a scenario could easily be included in the postscenario feedback as this provides the candidate with a review of their performance with the opportunity for reflection and video playback. After reading their article, we aim to incorporate training on medicolegal matters into our future courses and highlight the medicolegal aspects in the postscenario debriefing. In conclusion, we suggest that the practice of crisis scenarios in a high-fidelity simulation setting provides an opportunity to reduce error, improve patient safety and decrease the risk of litigation for anaesthetists.

\section{References}

1 White SM, Deacy N, Sudan S. Trainee anaesthetists' attitudes to error, safety and the law. Eur J Anaesthesio/ 2009; 26:463-468.

2 Phipps DL, Beatty PCW, Parker D, et al. Motivational influences on anaesthetists' use of practice guidelines. Br J Anaesth 2009; 102:768-774.

3 Chopra V, Gesink BJ, DeJong J, et al. Does training on an anaesthesia simulator lead to improvement in performance? Br J Anaesth 1994; 73:293-297.

4 Ericsson KA, Charness N. Expert performance, its structure and acquisition. Am Psychol 1994; 49:725-747.

5 Ericsson KA. Deliberate practice and the acquisition and maintenance of expert performance in medicine and related domains. Acad Med 2004; 79:S70-S81.

6 Kuduvalli PM, Parker CJR, Leuwer M, Guha A. Retention and transferability of team-resource-management skills in anaesthetic emergencies: the longerterm impact of a high-fidelity simulation-based course. Eur J Anaesthesiol 2009; 26:17-22.

7 Cumin D, Merry AF, Weller JM. Standards for simulation. Anaesthesia 2008; 63:1281-1287.

DOI:10.1097/EJA.0b013e328330ecbe

\section{Misinterpretation of a movable mass attached to the aortic valve imaged by transoesophageal echocardiography}

Ryoji lida, Yuko Kondo, Jitsu Kato and Setsuro Ogawa

From the Department of Anesthesiology, Nihon University School of Medicine, Tokyo, Japan

Correspondence to Ryoji lida, MD, PhD, Department of Anesthesiology, Nihon University School of Medicine, 30-1 Oyaguchi-Kamicho, Itabashi-Ku, Tokyo 173-0032, Japan

Tel: +81 33972 8111; fax: +81 33972 9448; e-mail: ryoiida@med.nihon-u.ac.jp

Received 18 September 2009 Accepted 19 September 2009

Editor,

Intraoperative transoesophageal echocardiography (TEE) is a useful tool not only for monitoring surgical patients but also for evaluating and diagnosing cardiac lesions during surgery and guiding surgical approaches. Several reports have described the incidental identification of cardiac lesions using TEE. ${ }^{1-3}$ This case alerts echocardiographers regarding the possible misinterpretation of an apparent movable cardiac mass.

A 76-year-old man was scheduled for coronary artery bypass graft (CABG) surgery. He was admitted to our hospital and diagnosed with acute myocardial infarction 2 months prior to the scheduled surgery. Coronary angiography revealed total occlusion in segments 1 and 6 and significant stenosis (90\%) in segment 13 . The total occlusion in segment 1 was alleviated by percutaneous coronary intervention, resulting in nonsignificant stenosis (25\%). Preoperative transthoracic echocardiography (TTE) revealed an ejection fraction of $67 \%$ with mild hypokinesis of the inferior wall, trivial mitral regurgitation and no abnormalities of the aortic valve. TEE was not performed preoperatively.

After induction of anesthesia for the CABG surgery, TEE (ACUSON-CV70, Siemens, Tokyo, Japan), which was equipped with two-dimensional imaging with colour flow 
Fig. 1

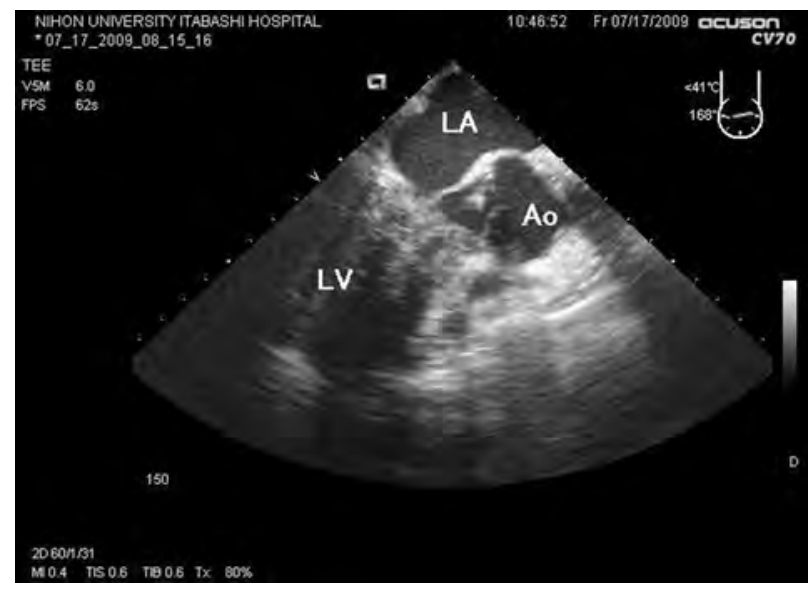

Midoesophageal modified long-axis view exhibited a movable mass-like object attached to the noncoronary cusp of the aortic valve. Ao, aorta; LA, left atrium; LV, left ventricle.

Doppler, was performed. A midoesophageal, modified long-axis view of the aortic valve revealed a $6.5 \mathrm{~mm} \times 3.5 \mathrm{~mm}$, high-echoic, mobile, pedunculated mass attached to the noncoronary cusp (Fig. 1 and Video Loop 1, http://links.lww.com/EJA/A1). The movement of the mass appeared to be synchronized with the aortic valve. Colour flow Doppler exhibited only trivial aortic regurgitation. The mass was visible in the wide range of the multiplane angle from the modified long-axis view to the true long-axis view of the aortic valve. A short-axis view of the aortic valve revealed mild hypertrophy of the noncoronary cusp at the level of the aortic valve (Fig. 2 and Video Loop 2, http://links.lww.com/EJA/A2). The view was unable to provide any clear images of the area

Fig. 2

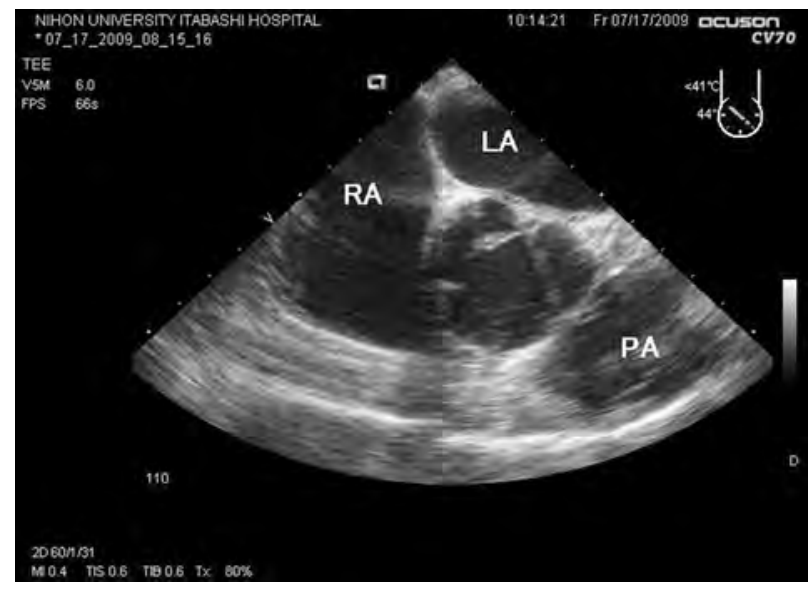

Midoesophageal short-axis view of the aortic valve exhibited mild hypertrophy of the noncoronary cusp. LA, left atrium; PA, pulmonary artery; RA, right atrium. proximal to the aortic valve due to the appearance of the basal left ventricle, and we were unable to detect the mass there. The movable mass was interpreted as likely representing a tumour, thrombus, vegetation or Lambl's excrescences. After detailed discussion with the cardiac surgeon regarding the movable mass and completion of distal anastomosis of CABG, the aorta was opened and the aortic valve was observed through the transaortic approach. Although no abnormal structures were attached to the aortic valve, part of the noncoronary cusp leaflet had yellowish degenerative bulging hypertrophy. After the aorta was closed and the cardiopulmonary bypass was completed, the aortic valve was again evaluated by TEE. The mass-like structure was still visible in the same view as it was during the pre-bypass period.

Accurate diagnosis of the mass-like structure was necessary in this case because a pedunculated, movable mass carries the risk of life-threatening complications such as stroke, embolism and acute valvular dysfunction. TEE is a useful imaging modality for assessment of movable intracardiac masses. With TEE, optimal high resolution and proximity between the transducer and the heart sometimes provide superior evaluation of the characteristics of a movable cardiac mass compared with TTE. It has been reported that a fibroelastoma attached to the aortic valve was incidentally identified with intraoperative TEE although it was not shown by preoperative TTE. ${ }^{2,3}$ However, in our case, the hypertrophic region of the aortic valve leaflet was misinterpreted as a movable cardiac mass. It was postulated that the bulging hypertrophic region of the noncoronary cusp was imaged only during diastole, this being interpreted as a movable mass during the cardiac cycle. Misinterpretation of cardiac lesions by TEE has been reported. ${ }^{4,5}$ A mobile mass on a prosthetic mitral valve observed by TEE was reportedly misinterpreted as vegetation when it was actually a pannus. ${ }^{4}$ In our case, the hypertrophic region of the valve appeared to be a mass-like structure that moved with the cardiac cycle, as viewed by TEE. This is one possible pitfall of TEE. It is extremely important, though sometimes difficult, to differentiate mass-like structures attached to the aortic valve. This case has an important clinical message for the interpretation of a movable mass on the aortic valve with TEE.

\section{References}

1 Huang $\mathrm{CH}, \mathrm{Wu}$ GJ, Yeh HM, et al. Incidental transesophageal echocardiographic findings of a mass on the aortic valve. J Cardiothorac Vasc Anesth 2004; 18:114.

2 Gopaldas RR, Atluri PV, Blaustein AS, et al. Papillary fibroelastoma of the aortic valve: operative approaches upon incidental discovery. Tex Heart Inst J 2009; 36:160-163.

3 Botta L, Dell'Amore A, Pilato E, et al. Papillary fibroelastoma of the aortic valve: incidental finding with intraoperative transesophageal echocardiography. Cardiovasc Pathol 2007; 16:59-60.

4 lonescu AA, Butchart EG. The vegetation that was not a vegetation: excusable misinterpretation of transesophageal echocardiography findings in late infective endocarditis of a prosthetic mitral valve. J Am Soc Echocardiogr 2000; 13:322-324. 
5 Huang $\mathrm{HH}$, Lin PL, Chao IF, et al. Misdiagnosed right atrial tumor identified by intraoperative transesophageal echocardiography. Cardiol J 2009; 16:175-176.

DOI:10.1097/EJA.0b013e3283333ade

\section{Alleged intraoperative awareness: a report of two cases}

Jiyeon Sim, Wonsik Ahn and Hyunsook Kim

From the Department of Anesthesiology, College of Medicine, University of Ulsan (JS) and Department of Anesthesiology and Pain Medicine, Seoul National University Hospital (WA, HK), Seoul, Republic of Korea

Correspondence to Wonsik Ahn, MD, Department of Anesthesiology and Pain Medicine, Seoul National University Hospital, 28 YongunDong Jongrogu, Seoul 110-744, Republic of Korea

Tel: +82 22072 3087; fax: +82 2747 5639; e-mail: aws@snu.ac.kr

Received 18 September 2009 Accepted 22 September 2009

Intraoperative awareness can traumatize those patients who experience it and result in anxiety disorders such as posttraumatic stress disorder. ${ }^{1}$ Although it occurs rarely, anaesthesiologists should examine anaesthetized patients carefully to detect its signs and try to prevent it. ${ }^{2-5}$ Most of the common causes of intraoperative awareness are the mechanical problems with vaporizers, anaesthetic machines or respiratory circuits. It has been postulated that changes in patients' blood pressure (BP) or heart rate caused by low concentrations of anaesthetics were concealed by the use of opioids under these circumstances. ${ }^{6}$ Similar situations may occur during emergence from anaesthesia if opioids are administered to reduce postoperative pain. Patients' anxiety or fear encountered during recovery, which can be magnified by the confused mental state, tends to be neglected. This distress, however, may be very serious and intense if patients confuse the memories during emergence from general anaesthesia with intraoperative awareness. We report two patients who encountered severe discomfort during recovery and asserted that they experienced intraoperative awareness.

\section{Case reports}

\section{Case 1}

A 69-year-old woman was diagnosed with stomach cancer and admitted for a subtotal gastrectomy. She was operated on for acute appendicitis under general anaesthesia 5 years ago. She also had ulcerative colitis and took acetic salicylic acid and prednisolone. No sedative or opioid premedication was given before surgery. When the patient arrived in the operating room, she was monitored with an electrocardiogram, a noninvasive arterial BP monitor, a pulse oximeter and a peripheral nerve stimulator. Anaesthesia was induced with thiopental $250 \mathrm{mg}$ and fentanyl $100 \mu \mathrm{g}$ intravenously. After endotracheal intubation was achieved following neuromuscular blockade, anaesthesia was maintained with sevoflurane and a mixture of oxygen and air. Concentrations of oxygen, inspired/end-tidal sevoflurane and carbon dioxide were measured with an anaesthetic machine (Primus; Drager, Lubeck, Germany) and patient monitor (Solar 8000M; GE Medical System, Milwaukee, Wisconsin, USA). The sevoflurane concentration was adjusted in the range of $2.2-3.9 \mathrm{vol} \%$ based on the haemodynamic response of the patient. Her vital signs were so stable during the operation with no signs of inadequate anaesthesia, such as hypertension, tachycardia, sweating and movement, that no more opioids were administered. At the end of the surgery, residual neuromuscular blockade was reversed with neostigmine mixed with atropine, and the patient's lungs were ventilated with $100 \%$ oxygen. The total anaesthesia time was $120 \mathrm{~min}$. Although she did not regain consciousness completely, we removed the endotracheal tube because the patient was breathing adequately and seemed very uncomfortable with it. After observing the patient carefully for a few minutes, we started patient-controlled analgesia [(PCA) morphine $40 \mathrm{mg}$, fentanyl $1500 \mu \mathrm{g}$, ondansetron $4 \mathrm{mg}$, isotonic saline $64 \mathrm{ml}$, total $100 \mathrm{ml}$, continuous infusion rate $1 \mathrm{ml} \mathrm{h}^{-1}$, bolus $0.5 \mathrm{ml}$ and lockout time $15 \mathrm{~min}$ ] to relieve her postoperative pain and transferred her to the postanaesthesia care unit (PACU). Because she had not recovered consciousness after about an hour in the PACU, the PCA was stopped, and, thereafter, she became fully awake. After staying in the PACU for another $2 \mathrm{~h}$ without any particular complaint, she was transferred to a general ward. However, the next day, she insisted that she had experienced intraoperative awareness. We visited her and asked her about it. She told us that there were other patients beside her, and the ceiling light was different from the one she had seen during the induction of anaesthesia. The place she remembered must have been the PACU, not the operating room, so we explained that she had confused the two places and assured her of the adequate depth of anaesthesia during surgery. However, she disagreed with us and asserted that she was awake during surgery. She said that she would refuse to be operated on again under general anaesthesia because of the horrible experience of intraoperative awareness.

\section{Case 2}

An 81-year-old woman was admitted for a subtotal gastrectomy. She had undergone a repair surgery of the knee cartilage under spinal analgesia 2 years earlier. She had a history of hypertension and diabetes mellitus and took amlodipine, candesartan and glimepride. Anaesthesia was induced with thiopental $250 \mathrm{mg}$ and fentanyl $100 \mu \mathrm{g}$ intravenously, and vecuronium $8 \mathrm{mg}$ was administered. After endotracheal intubation, anaesthesia was maintained with desflurane, oxygen and air. The inspired and endtidal concentrations of oxygen, desflurane and carbon dioxide were monitored. The desflurane concentration was changed between 4.3 and $6.0 \mathrm{vol} \%$ to maintain a sufficient depth of anaesthesia to prevent sympathetic 
responses by the patient. No additional opioids were required during surgery. The total anaesthesia time was $220 \mathrm{~min}$. After the operation was over, neuromuscular blockade was reversed with neostigmine mixed with atropine, and $100 \%$ oxygen was supplied. When the patient was breathing adequately and responded to verbal commands, the endotracheal tube was removed. We started PCA (morphine $30 \mathrm{mg}$, fentanyl $1000 \mu \mathrm{g}$, ondansetron $4 \mathrm{mg}$, isotonic saline $25 \mathrm{ml}$, total $50 \mathrm{ml}$, continuous infusion rate $1 \mathrm{ml} \mathrm{h}^{-1}$, bolus dose $0.5 \mathrm{ml}$ and lockout time $15 \mathrm{~min}$ ) for the relief of pain and transferred the patient to surgical ICU (SICU). She did not regain her consciousness completely until $3 \mathrm{~h}$ after her arrival at SICU and occasionally complained of pain. After she became fully awake, she did not mention any discomfort except postoperative pain and was transferred to a general ward the next day. We heard that she insisted that she had experienced intraoperative awareness and visited her. She told us that she awoke from anaesthesia because of pain during the operation and had asked us to reduce her pain. She felt that she was not given enough analgesics, although a nurse had explained to her that more analgesics had been given. She must have confused SICU and the operating room based on communication with a nurse, and we gave her a full explanation to clear up any misunderstandings. However, she refused to accept our explanation and said that she would never be operated on again under general anaesthesia for fear of awareness during surgery.

\section{Discussion}

The subconscious recollection of intraoperative events could be excluded based on the end-tidal inhalant level and recall situation such as a different ceiling light and communication with a nurse.

There was one similar case report to ours. ${ }^{7}$ A 60 -year-old woman underwent a left colectomy under general anaesthesia with isoflurane and $\mathrm{N}_{2} \mathrm{O}$. After the operation was over, she was transferred to the PACU. It was decided to keep the endotracheal tube in place for some time because the patient was very obese and had a history of obstructive sleep apnoea. Morphine was administered intermittently, and the endotracheal tube was removed after $4 \mathrm{~h}$. The next day, the patient complained of awareness during the operation and refused all further operations, although the authors explained the situation.

In the course of speculation about the causes of patients' confusion, we have found some similarities between our cases and this one. First, opioid-containing analgesia was started even before the patients regained consciousness fully. Second, it took the patients a few hours to become fully awake, and they complained of intraoperative awareness on the first postoperative day. Finally, all of them were probably elderly Asian women. Although the report by $\mathrm{Ho}^{7}$ did not describe the race of the patient, it was reported in Hong Kong, and she was likely to be an Asian. Considering all these facts, immediate postoperative administration of opioids, along with the patients' old age and the severe pain during a slow recovery of consciousness, could have an influence on their confusion. It might be affected by the racial differences because there was no report on white patients.

Attention should be paid to discriminate between these situations and postoperative delirium, which occurs especially in older patients. Anticholinergic agents, such as atropine and glycopyrrolate, are the precipitating factors for delirium after surgery. However, our patients were alert and did not meet the criteria of postoperative delirium, which include confusions, hallucinations and cognitive disorders such as disorientation and memory disorder.

Although two big awareness studies ${ }^{5,6}$ analysed multiple aspects of awareness, they did not consider discomfort during recovery. The anxiety and distress encountered during recovery from anaesthesia can be confused with intraoperative awareness and can cause patients severe psychological stress similar to real intraoperative awareness. We suggest that more attention should be paid to postoperative alleged awareness in the elderly patients who receive opioids during the recovery period.

\section{References}

1 Mashour GA, Jiang YD, Osterman J. Perioperative treatment of patients with a history of intraoperative awareness and posttraumatic stress disorder. Anesthesiology 2006; 104:893-894.

2 Sebel PS, Bowdle TA, Ghoneim MM, et al. The incidence of awareness during anesthesia: a multicenter United States study. Anesth Analg 2004; 99:833-839.

3 Davidson AJ, Huang GH, Czarnecki C, et al. Awareness during anesthesia in children: a prospective cohort study. Anesth Analg 2005; 100:653-661.

4 Kakinohana M, Nakamura S, Miyata Y, Sugahara K. Emergence from propofol anesthesia in a nonagenarian at a bispectral index of 52. Anesth Analg 2005; 101:169-170.

5 Pollard RJ, Coyle JP, Gilbert RL, Beck JE. Intraoperative awareness in a regional medical system: a review of 3 years' data. Anesthesiology 2007; 106:269-274.

6 Bergman IJ, Kluger MT, Short TG. Awareness during general anaesthesia: a review of 81 cases from the anaesthetic incident monitoring study. Anaesthesia 2002; 57:549-556.

7 Ho AM. 'Awareness' and 'recall' during emergence from general anaesthesia. Eur J Anaesthesiol 2001; 18:623-625.

DOI:10.1097/EJA.0b013e32833371c6

\section{General anaesthesia for a patient with Williams-Beuren syndrome: is sevoflurane and ketamine contraindicated?}

Laetitia Hélaine and Gabor Kiss

From the Department of Anaesthesia and Surgical Intensive Care, University Hospital of La Cavale Blanche Brest, Brest Cedex, France

Correspondence to Gabor Kiss, Department of Anaesthesia and Surgical Intensive Care, University Hospital of La Cavale Blanche Brest, Brest Cedex 29609, France

Fax: +33 2983474 41.e-mail: gaborkiss2001@hotmail.com

Received 22 September 2009 Accepted 28 September 2009 
Literature is contradictional on the utilization of volatile anaesthetics and contraindicates ketamine in patients with Williams-Beuren syndrome (WBS). In our patient, the use of sevoflurane and ketamine at analgesic doses was unavoidable but beneficial. WBS is a rare genetical disorder linked with an increased risk of mortality during anaesthesia. We describe the case of a 38-year-old male patient with WBS who underwent thoracic surgery under general anaesthesia for pleural abrasion and pleural talcage to treat recurring spontaneous pneumothorax. A preoperative echocardiography revealed moderate aortic valve stenosis. Because the mentally retarded patient was too agitated to allow the insertion of a peripheral venous cannula, gas induction was performed with sevoflurane. Following, the patient was intubated with a double lumen endotracheal tube in order to exclude the right bronchus from ventilation. Anaesthesia was maintained with oxygen/air/sevoflurane. In spite of a possible link between WBS and malignant hyperthermia, uncertainty in the current literature does not exclude the use of volatile agents in these patients. Also, substances with vagolytic effects, such as ketamine should be avoided. In our case, utilization of a small analgesic dose of ketamine and sevoflurane was beneficial and the patient remained haemodynamically stable during and after the operation.

\section{Introduction}

Williams-Beuren syndrome (WBS) is a rare genetic disorder (one birth in 20000) characterized by facial and severe cardiovascular malformation (supravavular aortic stenosis and/or pulmonary artery stenosis) and mental retardation. This is due to chromosomial microdeletion on $7 \mathrm{q} 11.23$ that involves several genes such as the one responsible for the expression of elastine. ${ }^{1}$

Patients with this syndrome have a significant increase for myocardial ischaemia and a higher frequency of sudden death if under general anaesthesia or sedation. ${ }^{1}$

\section{Case report}

We describe the case of a 38-year-old man who has WBS with moderate aortic stenosis and mental retardation. He had no other past medical history. The patient underwent surgery for pleural talcage under general anaesthesia for recurring right pneumothorax.

On the day of the operation, he received a premedication with $5 \mathrm{mg}$ of midazolam and $100 \mathrm{mg}$ of metoprolol orally $2 \mathrm{~h}$ prior to surgery. On arrival in the operating theatre, the patient was agitated and it was impossible to insert an intravenous line without sedation. Therefore, gas induction with $6 \%$ of sevoflurane in oxygen was performed in order to establish an intravenous access. An ECG with a $\mathrm{CM}_{5}$ lead configuration was applied once agitation subsided during gas induction. Then, $80 \mathrm{mg}$ of propofol, $20 \mu \mathrm{g}$ of sufentanyl, $25 \mathrm{mg}$ of atracurium and $60 \mathrm{mg}$ of lidocaine were injected. Orotracheal intubation was per- formed with a double lumen tube in order to exclude the right lung during surgery. Anaesthesia was maintained with oxygen/air and sevoflurane with an additional bolus of $10 \mu \mathrm{g}$ of sufentanyl. Five milligrams of ketamine was infused during $15 \mathrm{~min}$ to provide postoperative analgesia. Mean arterial blood pressure remained stable at around $70 \mathrm{mmHg}$ during the whole procedure. During exclusion of the right lung, the lowest oxygen saturation measured was $95 \%$. Surgery lasted for $40 \mathrm{~min}$ and extubation was smooth.

One hour after the surgery, the patient was discharged from the recovery room and transferred to the cardiothoracic intensive care unit where recovery was uneventful. He was sent back to the ward the following day.

\section{Discussion}

WBS has first been described by J.C. Williams in 1961 and A.J. Beuren in 1962 in patients presenting an association of supravalvular aortic stenosis and other symptoms. ${ }^{2,3}$ The facial abnormalities are characterized by the presence of a flat nasal base, bulbous extremities, buccodental abnormalities, a large mouth with bulging large lower lips, a long philtrum, big cheeks, periorbital oedema, epicanthus and sometimes stellar iris. Current literature states that these facial abnormalities are not linked to difficulties in airway management or oral-tracheal intubation, as neither was the case in our patient. However, other problems are frequent in adult WBS patients: ophthalmological problems, hypothyroidism, growth retardation, glucose intolerance and joint problems. As was the case in our patient, there is also a characteristic neuropsychological profile with the presence of a cognitive deficit, paradoxale use of the language and hypersociability.

There are several reports in medical literature on sudden death in WBS patients who underwent general anaesthesia or sedation. Cardiac arrest of ischaemic origin was often preceded by arterial hypotension combined with bradycardia. One series of autopsies revealed that 14 out of 15 WBS patients had abnormal coronary arteries. ${ }^{1}$ Often, this was associated with supravalvular aortic stenosis and pulmonary artery stenosis. Apart from moderate aortic stenosis, no other cardiac malformations were present in our patient.

Preoperative check-up of the WBS patient should include an electrocardiogram and echocardiography to evaluate the presence of aortic stenosis. Coronarography can be requested if major haemodynamic variations are expected during surgery. On the contrary, coronarography in a WBS patient with mental retardation might necessitate sedation and therefore benefit should outweigh risk.

One case report of a patient who presented masseter spasm under general anaesthesia had raised the suspicion that WBS is associated with malignant hyperthermia. ${ }^{4}$ Indeed, the association between WBS and malignant 
hyperthermia seems plausible because of the proximity of the chromosomial deletion responsible for WBS and the gene CACNL2A coding for a voltage-dependent calcium channel with mutants that are involved in malignant hyperthermia. Meanwhile, a recent case report ${ }^{5}$ has described a suspected link between postoperative malignant hyperthermia and WBS. On the contrary, a study by Mammi et al. ${ }^{6}$ has shown that the locus of CACNL2A is outside the deleted zone of chromosome 7 in WBS. Currently, literature does not provide a clear response about the use of volatiles for general anaesthesia in WBS patients. Several authors reported no complications after use of volatiles in these patients. ${ }^{7}$ Therefore, in this context and because of the agitation, a volatile gas induction was justified in our patient. The advantages of ischaemic preconditioning (IPC) with volatile agents for coronary patients are well accepted. Cardiac protection by IPG against the consequences of ischaemic reperfusion such as myocardial ischaemia, postoperative myocardial dysfunction and arrhythmia could be beneficial for the WBS patient with coronary abnormalities who are not always having a full cardiac preoperative check-up. Our patient had a normal sinus rhythm and did not have any signs of intraoperative and postoperative myocardial ischaemia. In case of the event of intraoperative supraventricular tachycardia, cardioversion should be the preferred treatment option. Vagolytics such as ketamine should be avoided. Nevertheless, it is also important to treat acute postoperative pain, which is a potential source of tachycardia. Therefore, we used ketamine in our patient at an analgesic dose of $0.1 \mathrm{mg} / \mathrm{kg}$, which does not have any vagolytic effects at this dosage. This also allows decreasing the incidence of postoperative complications in thoracic surgery that are indirectly caused by pain such as hypoxia and pneumonia.

As WBS patient have ventricular hypertrophy and do not tolerate a rapid rise in intraventricular volume, it is also important to pay attention to the left ventricular precharge in order to avoid sudden death. Consequently, fluids were administered slowly and limited to $500 \mathrm{ml}$ of normotonic Ringer solution perioperatively.

\section{Conclusion}

In spite of a possible link between WBS and malignant hyperthermia, literature does currently not provide a clear response about the use of volatiles for general anaesthesia in WBS patients. As several authors reported no complications after the use of volatiles in these patients and because the mentally retarded patient was too agitated to allow the insertion of a peripheral venous cannula, a volatile gas induction and maintenance with oxygen/air/sevoflurane was justified. It is also advised in WBS patients that substances with vagolytic effects, such as ketamine should be avoided. However, we used ketamine at an analgesic dose of $0.1 \mathrm{mg} / \mathrm{kg}$, which does not have any vagolytic effects and achieved good postoperative analgesia without any haemodynamic abnormalities.

\section{Acknowledgement}

We are grateful to Jean-Pierre Salou, anaesthetic nurse at the University Hospital in Brest (France), who helped us to constitute and analyse the patient's notes.

\section{References}

1 Burch TM, McGowan FX Jr, Kussman BD, et al. Congenital supravalvular aortic stenosis and sudden death associated with anesthesia: what's the mystery? Anesth Analg 2008; 107:1848-1854.

2 Williams JC, Barratt-Boyes BG, Lowe JB. Supravalvular aortic stenosis. Circulation 1961; 24:1311-1318.

3 Beuren AJ, Apitz J, Harmjanz D. Supravalvular aortic stenosis in association with mental retardation and a certain facial appearance. Circulation 1962; 26:1235-1240.

4 Patel J, Harrison MJ. Williams syndrome: masseter spasm during anaesthesia. Anaesthesia 1991; 46:115-116.

5 Kohase H, Wakita R, Doi S, Umino M. General anesthesia for dental treatment in a Williams syndrome patient with severe aortic and pulmonary valve stenosis: suspected episode of postoperatively malignant hyperthermia. Oral Surg Oral Med Oral Pathol Oral Radiol Endod 2007; 104:e17-e20.

6 Mammi I, lles DE, Smeets D, et al. Anesthesiologic problems in Williams syndrome: the CACNL2A locus is not involved. Hum Genet 1996; 98:317320.

7 Andrzejowski J, Mundy J. Anaesthesia for MRI angiography in a patient with Williams syndrome. Anaesthesia 2000; 55:97-98.

DOI:10.1097/EJA.0b013e32833393c7 